\title{
La tuberculose bovine à Madagascar
}

\author{
par J. BLANCOU, C. RORHBACH. A. PERDRIX, \\ P. CHOQUEL, G. ROSNER (*)
}

\begin{abstract}
RESUME
Une enquête sur la tuberculose bovine a été réalisée à Madagascar, en 1969-1970, par les méthodes d'inspection d'abattoir et de tuberculination intra-dermique. La proportion des saisies pour tuberculose, effectuées dans tous les abattoirs de TlTle au cours des dix dernières années, a été relevée et contrôlée par sondages régionaux : 21 p. 100 des 1.465 .000 bovins inspectés ont été reconnus tuberculeux. Les résultats de 9.217 tuberculinations effectuées ont êté analysés par race, sexe, âge et mode d'élevage des bovins éprouvés. Les solutions actuellement possibles à Madagascar pour abaisser le taux de tuberculose bovine sont envisagées.
\end{abstract}

\section{INTRODUCTION}

Si l'Ile de Madagascar a un taux d'endémie tuberculeuse humaine moyen (6), il n'en est pas de même de l'enzootic animale, comme l'ont déjà précisé plusieurs travaux $(5,7) . \mathbf{L a}$ maladie a pris naissance, au début du siècle semble-t-il, dans le sud de l'Ile. Depuis son apparition, son développement a été sujvi surtout d'après les relevés de saisies pour tuberculose effectuées dans les abattoirs, les tuberculinations s'étant toujours avérées d'interprétation statistique délicate.

En 1969-1970, une vaste opération intitulée «Lutte contre la mortalité des veaux - éradication de la tuberculose "a été financée par la République Malgache et confiée à l'Institut d'élevage et de médecine vétérinaire des pays tropicaux. Le projet concernant la tuberculose prévoyait en particulier " une définition des principes et méthodes d'éradication de la tuberculose dans la zone de desserte des abattoirs de Majunga et Tananarive ».

(*) Institut d'Elevage et de Médccine vétérinaire des Pays tropicaux. Région de recherches de Madagascar. B.P. n० 862
Pour cela, il a été réalisé une enquête préliminaire approfondie de l'enzootie dans les régions désignées, par zone, mode d'élevage, âge et race. Ce sont les méthodes employées dans cette enquête, les résultats obtenus et les conclusions qu'ils nous suggèrent qui sont l'objet de la présente étude.

\section{METHODES D'ETUDE}

Trois méthodes d'étude sont théoriquement applicables dans ce type d'enquête :

1. Inspection d'abattoirs, avec relevé détaillé des lésions tuberculeuses observées.

2. Tuberculination des animaux.

3. Examen clinique des animaux.

Nous rapportons en détail la mise en ouvre de ces trois méthodes, leurs avantages et leurs inconvénients.

\section{INSPECTION D'ABATTOIRS}

\section{Méthodes actuelles d'inspection des viandes à Madagascar}

L'inspection des viandes est assurée actuellement, dans les principaux centres de consom- 
mation, par des assistants ou adjoints techniques du Service de l'élevage ayant reçu une formation appropriée au cours de lcurs ćtudes. En leur absence (cas des centres dépourvus de poste vétérinaire), ce sont les médecins formés à l'école nationale de médecine de Tananarive qui assurent cette inspection, pour laquelle ils ont reçu également un enseignement spécialisé.

Les résultats de ces inspections sont consignés dans Ies rapports de chaque poste vétérinaire et centralisés ensuite à Tananarive. Sur le plan statistique, ces résultats comportent malheureusement deux graves inconvénients :

1. Ils concernent presque uniquement des bœufs de boucherie (c'est-à-dire des animaux âgés de plus de 5 ans en général).

2. N'étant pas relevés sur des cahiers d'un modèle standard, ils comportent rarement les indications indispensables d'âge, et origine des animaux abattus (lesquels viennent parfois de régions éloignées de plus de 500 kilomètres). Plusieurs saisies, concernant le même animal, peuvent être reportées sur le cahier sans que cela soit explicité.

Néanmoins, la masse totale de ces documents constitue une information peu suspecte de pécher par excès : nous reproduisons dans un tableau général les statistiques que nous avons relevées concernant les différentes provinces au cours des dix dernières années.

\section{Méthode employée dans notre enquête}

Dans notre enquête, nous avons accordé beaucoup moins d'importance à l'inspection des viandes qu'à la tuberculination, puisqu'elle était d'avance sujette aux critiques précédemment formulées contre l'inspection traditionnelle. Si nous l'avons cependant utilisée, c'était surtout dans le but de réaliser un sondage sur la valeur de cette inspection traditionnelle. Les résultats de cette enquête ont été notés sur une fiche d'un modèle reproduit en annexe, établi sur les conseils des bureaux d'études d'International Business Machine, établissement chargé de leur exploitation ultérieure.

Ces fiches indiquent donc, pour chaque catégorie de sujets, la proportion des lésions affectant soit le système ganglionnaire, soit le parenchyme pulmonaire, soit un autre viscère, soit la carcasse ou ses séreuses pariétales.

\section{TUBERCULINATIONS}

\section{Méthode actuelle de tuberculination à Madagascar}

Si plus de 30.000 doses de tuberculine pour réaction intradermique sont demandées chaque année au Laboratoire central de l'élevage (qui en assure la fabrication), les résultats des tuberculinations effectuées ne sont pas tous rapportés.

Ces tuberculinations n'étant pas réalisées dans un but d'information statistique, nous avons pu difficilement les exploiter. En effet :

1. Ces tuberculinations concernent presque uniquement des troupeaux de zones urbaines ou suburbaines, en stabulation, ou des troupeaux de bêtes de boucherie contrôlées pour l'exportation.

2. Elles comportent très rarement les indications indispensables à leur analyse (race, âge, sexe, mode d'élevage en particulier).

\section{Méthodes employées dans notre enquête}

\section{Technique}

Nous avons utilisé la technique de l'intradermo réaction classique, pratiquée au tiers moyen de l'encolure, avec une tuberculine fabriquée au Laboratoire central de l'élevage.

Cette tuberculine est fabriquée selon les techniques classiques, sur milieu de Sauton, à partir d'une souche de bacille bovin isolée à Madagascar. Elle titre 2.500 U.I. par ml.

La dose injectée varie de 0,1 à $0,2 \mathrm{ml}$, la lecture de la réaction étant faite 48 à 72 heures plus tard: seules les réactions nettement positives ont été prises en considération. Les animaux tuberculinés étaient marqués par rasage des poils de l'encolure, pour éviter toute substitution d'animaux lors de la visite de contrôle. Toutes les injections et lecture des réactions ont été faites par des vétérinaires. Les animaux étaient rassemblés le plus souvent dans des couloirs de vaccination de fabrication traditionnelle : parcs en branchages, fonds de ravins clôturés de branches, anciens murs de fabrication.

\section{Choix des sujets à tuberculiner}

Compte tenu de l'intégration de l'enquête dans une opération beaucoup plus vaste de déparasitage systématique des veaux, nous 
n'avons pas utilisé les méthodes de sondages aléatoires recommandées en pareil cas. Les troupeaux choisis n'ont pas été tirés au sort : c'étaient ceux dont l'accès était favorisé par les opérations de déparasitage, le troupeau choisi étant alors entièrement tuberculiné.

Notre enquête a donc une prétention très limitée : désigner les catégories de bovins les plus atteints dans chaque zone, et non dresser une carte de répartition de la maladie.

\section{Zones d'élevage étudiées}

La « zone de desscrte des abattoirs de Tananarive et Majunga " englobe deux régions géographiques distinctes:

Hauts Plateaux et Ouest. Ces deux régions peuvent se caractériser ainsi :

- Hauts Plateaux : cette zone comprend les "hautes terres» proprement dites et le "Moyen-Ouest ". Ce dernier est une pénéplaine primaire, de 7 à 900 mètres d'altitude, de climat assez froid et humide $(1.655 \mathrm{~mm}$ de pluie et $22,3^{\circ}$ de moyenne à Tsiroanomandidy). Son sol ferralitique, couvert d'une riche savane herbacée, en fait une région de prédilection pour l'embouche des bovins nés dans l'Ouest. La population est d'ethnie Merina en majorité, associant souvent agriculture et élevage avec de bons résultats.

- Ouest : Pénéplaine sédimentaire, de basse altitude, bordant la côte. Elle contraste avec la précédente par son relief moins tourmenté et son climat tropical chaud. L'enquête couvrait trois zones administratives distinctes dans cette région: Morondava, Majunga et Antsohihy, pour lesquelles les moyennes de température et pluviométrie annuelles sont respectivement de $24,3^{\circ}-743 \mathrm{~mm}, 26,4^{\circ}-1.567 \mathrm{~mm}$ et $27^{\circ}-1.493 \mathrm{~mm}$. Sol sableux ou argilo-sableux assez pauvre, de même que sa couverture herbacée : c'est une région à vocation " naisseuse " qui envoie des bovins dans presque toute l'Ile. Population Sakalava ou Tsimihety, éleveurs traditionnels.

\section{Méthode de relevé et d'exploitation des résultats}

Le résultat de la tuberculination, relevé sur fiche du modèle reproduit en annexe, comporte donc une définition complète de l'animal. Ces fiches, triées ensuite par des procédés électroniques, peuvent donner théoriquement toutes les combinaisons demandées. C'est ainsi qu'il est possible de déterminer que 22,1 p. 100 des zébus malgaches mâles, âgés de 3 à 10 ans, élevés en liberté totale, ont réagi à la tuberculine lors de l'enquête faite dans la zone de Morondava. En fait, nous ne reproduirons ici que les résultats globaux de l'enquête dans chaque zone.

Il est très important de souligner que, si 9.217 résultats ont été relevés au total, plus de 12.000 tuberculinations ont été effectuées en réalité pour les obtenir. En effet une forte proportion d'animaux ne sont pas représentés à la visite de contrôle. Ce fait, déjà souligné en Afrique et à Madagascar $(1,2,7)$, reste le plus grave obstacle à l'application de cette méthode de diagnostic en particulier et à l'éradication de la tuberculose en général, si elle se base sur l'élimination des réagissants. En conclusion, cette méthode reste imparfaite, surtout dans les conditions où elle fut obligatoirement utilisée : les résultats ont le seul mérite d'intéresser toutes les catégories de sujets et non pas les seuls animaux de boucherie.

\section{EXAMENS CLINIQUES}

Il existait peu de rapports sur la tuberculose clinique en élevage extensif, aussi espérionsnous combler cette lacune au cours de notre enquête. Or, de l'avis général, les examens cliniques dans les conditions imposées par l'organisation générale de l'opération se sont avérés beaucoup trop délicats : difficultés de distinguer maigreur physiologique et pathologique en saison sèche, essoufflement des animaux rassemblés dans les parcs, absence de commémoratifs, etc. Nous avons donc remplacé, au cours de l'enquête, ces examens par un interrogatoire précis des éleveurs concernant l'évolution de la maladie dans leur troupeau, et leur attitude envers elle : ces opinions sont rapportées dans le chapitre "discussion".

\section{RESULTATS}

\section{EXPOSE DES RESULTATS}

\section{INSPECTIONS D'ABATTOIRS}

Nous distinguerons deux catégories de résultats :

- ceux du relevé général des saisies effectuées 
TABLEAU $\mathrm{N}^{\circ}$ I

Pourcentage de saisies effectuées pour tuberculose pulmonaire chez les bovins au cours des dix dernières années à Madagascar.

(Intervalle de confiance 1 p.100. Le chiffre suivant le nom de la localitê est reportế sur la carte de Madagascar, ci-contre).

\begin{tabular}{|c|c|c|c|c|c|}
\hline $\begin{array}{l}\text { l'rovince de } \\
\text { Diego-Suarez }\end{array}$ & $\begin{array}{c}\text { Province de } \\
\text { Tul êar }\end{array}$ & $\begin{array}{l}\text { Province de } \\
\text { Fianarantsoa }\end{array}$ & $\begin{array}{l}\text { Province de } \\
\text { Tananarive }\end{array}$ & $\begin{array}{l}\text { Province de } \\
\text { Majunga }\end{array}$ & $\begin{array}{c}\text { Province de } \\
\text { Tamatave }\end{array}$ \\
\hline $\begin{array}{l}\text { Diêgo-Suarez : I } \\
\frac{10.962}{157.818}=6,9\end{array}$ & $\begin{array}{l}\text { Tuléar* } \\
\frac{26.963}{209.303}=12,9\end{array}$ & $\begin{array}{l}\text { Fianarantsoa : } 53 \\
\frac{7.133}{41.436}=17,2\end{array}$ & $\begin{array}{l}\text { Tananarive }{ }^{* *}: 32 \\
\frac{116.798}{524527}=22,3\end{array}$ & $\begin{array}{l}\text { Majunga } \quad: 12 \\
\frac{25.272}{43.620}=58\end{array}$ & $\begin{array}{l}\text { Tamatave } \\
\frac{52.805}{125.854}=42\end{array}$ \\
\hline $\begin{array}{c}\text { Antalaha } \\
\frac{875}{9397}=9,3\end{array}$ & $\begin{array}{r}\text { Fort-Dauphin: } 47 \\
\frac{892}{13.833}=6,4\end{array}$ & $\begin{array}{cl}\text { Ambositra } & : 50 \\
\frac{1.456}{18.529}= & 7,8\end{array}$ & $\begin{array}{l}\text { Antsirabe } \quad: 38 \\
\frac{5 \cdot 257}{23.673}=22,2\end{array}$ & $\begin{array}{l}\text { Antsohihy } \quad: 10 \\
\frac{4.443}{20.761}=21,4\end{array}$ & $\begin{array}{c}\text { Ambatondrazaka }=24 \\
\frac{3.663}{11.465}=32\end{array}$ \\
\hline $\begin{array}{c}\text { Ambilobe } \\
\frac{546}{8623}=6,3\end{array}$ & $\begin{array}{l}\text { Morondava } \\
\frac{1020}{13080}=7,8\end{array}$ & $\begin{array}{c}\text { Manakara } \\
\frac{3030}{12.823}=23,6\end{array}$ & $\begin{array}{l}\text { Tsiroanomandidy: } 34 \\
\frac{5408}{13.239}=40,9\end{array}$ & $\begin{array}{l}\text { Maintirano : } 18 \\
\frac{3047}{6856}=44,4\end{array}$ & $\begin{array}{c}\text { Mananara } \\
\frac{423}{1372}=30,8\end{array}$ \\
\hline $\begin{array}{c}\text { Vohêmar } \quad: 3 \\
\frac{477}{7786}=6,1\end{array}$ & $\begin{aligned} & \text { Betroka }: 44 \\
& \frac{239}{3592}=6,6\end{aligned}$ & $\begin{array}{l}\text { Thosy } \\
\frac{2408}{11574}=20,8\end{array}$ & $\begin{array}{c}\text { Arivonimamo }: 30 \\
\frac{396}{8397}=4,7\end{array}$ & $\begin{array}{l}\text { Ambato-Boeni : } 15 \\
\frac{1453}{5251}=27,7\end{array}$ & $\begin{array}{c}\text { Maramanga } \\
\frac{334}{1144}=29,26\end{array}$ \\
\hline $\begin{array}{l}\text { Ambanja } \quad: 5 \\
\frac{2}{5839}=41,5\end{array}$ & $\begin{array}{cl}\text { Ankazoabo } & : 43 \\
\frac{184}{2054}= & 9\end{array}$ & $\begin{array}{cl}\text { Andranalina } & : 58 \\
\frac{828}{8944}= & 9,2\end{array}$ & $\begin{array}{c}\text { Man jakandriana: } 33 \\
\frac{683}{7271}=9,4\end{array}$ & $\begin{array}{l}\text { Mandritsara : } 13 \\
\frac{1659}{4437}=37,4\end{array}$ & $\begin{array}{c}\text { Fênërive } \quad: 23 \\
\frac{297}{1113}=26,7\end{array}$ \\
\hline $\begin{array}{l}\text { Nossi-Be } \quad: 4 \\
\frac{1817}{5680}=32\end{array}$ & $\begin{array}{c}\text { Ampanihy } \\
\frac{70}{1764}=4\end{array}$ & $\begin{array}{c}\text { Ambohimahasoa: } 52 \\
\frac{587}{5986}=9,8\end{array}$ & $\begin{array}{c}\text { Andramasina }: 37 \\
\frac{218}{7102}=3\end{array}$ & $\begin{array}{c}\text { Analaiava }: 9 \\
\frac{969}{4359}=22,3\end{array}$ & $\begin{array}{c}\text { Brickaville } \quad: 27 \\
\frac{70}{873}=8\end{array}$ \\
\hline $\begin{array}{c}\text { Sambava } \\
\frac{340}{3909}=8,7\end{array}$ & $\begin{array}{c}\text { Ambovombe } \\
\frac{66}{1464}=4,5\end{array}$ & $\begin{array}{c}\text { Mananjary } \\
\frac{851}{4084}=20,8\end{array}$ & $\begin{array}{l}\text { Soavinandriana: } 35 \\
\frac{1414}{6793}=20,9\end{array}$ & $\begin{array}{c}\text { Befandriana : } 11 \\
\frac{586}{321}=15,7\end{array}$ & $\begin{array}{c}\text { Sainte-Marie } \quad: 21 \\
\frac{88}{799}=11\end{array}$ \\
\hline $\begin{array}{cc}\text { Andapa } & : 7 \\
\frac{137}{2046}=6,7\end{array}$ & $\begin{array}{c}\text { Mahabo* } \quad: 41 \\
\frac{533}{1240}=43\end{array}$ & $\begin{array}{cl}\text { Fandriana } & : 51 \\
\frac{270}{3706}= & 7,3\end{array}$ & $\begin{array}{l}\text { Ambatolampy }: 36 \\
\frac{185}{6057}=3\end{array}$ & $\begin{array}{l}\text { Marovoay** }: 14 \\
\frac{406}{2082}=19,5\end{array}$ & $\begin{array}{c}\text { Maroantsetra }: 19 \\
\frac{212}{678}=31,3\end{array}$ \\
\hline & $\begin{array}{r}\text { Belo/Tsirib : } 39 \\
\frac{145}{960}=15,1\end{array}$ & $\begin{array}{l}\text { Ambatofinand.: } 43 \\
\frac{281}{2295}=12,2\end{array}$ & $\begin{array}{l}\text { Ambohidratrimo: } 31 \\
\frac{799}{5437}=14\end{array}$ & $\begin{array}{l}\text { Tsaratanana : } 16 \\
\frac{803}{\operatorname{T948}}=41\end{array}$ & $\begin{array}{c}\text { Andilamena }: 22 \\
\frac{60}{377}=15,9\end{array}$ \\
\hline & $\begin{array}{l}\text { Autres abattoirs } \\
\qquad \frac{541}{17.621}=12\end{array}$ & $\begin{array}{l}\text { Autres abattoirs } \\
\qquad \frac{1722}{17621}: 9,77\end{array}$ & $\begin{array}{l}\text { Autres abattoirs } \\
\qquad \frac{1703}{13.586}=12,53\end{array}$ & $\begin{array}{l}\text { Autres abattoirs } \\
\frac{5}{17} \frac{141}{642}=29,14\end{array}$ & $\begin{array}{l}\text { Autres abattoirs } \\
\qquad \frac{62}{472}=13,13\end{array}$ \\
\hline
\end{tabular}

Total gếnêral :

305359 saisies pour tuberculose pulmonaire sur 1465338 bovins inspectés (soit 20,83 p.100)

* Fiabilité satisfaisante; ** Chiffres probablement sous-estimês. 


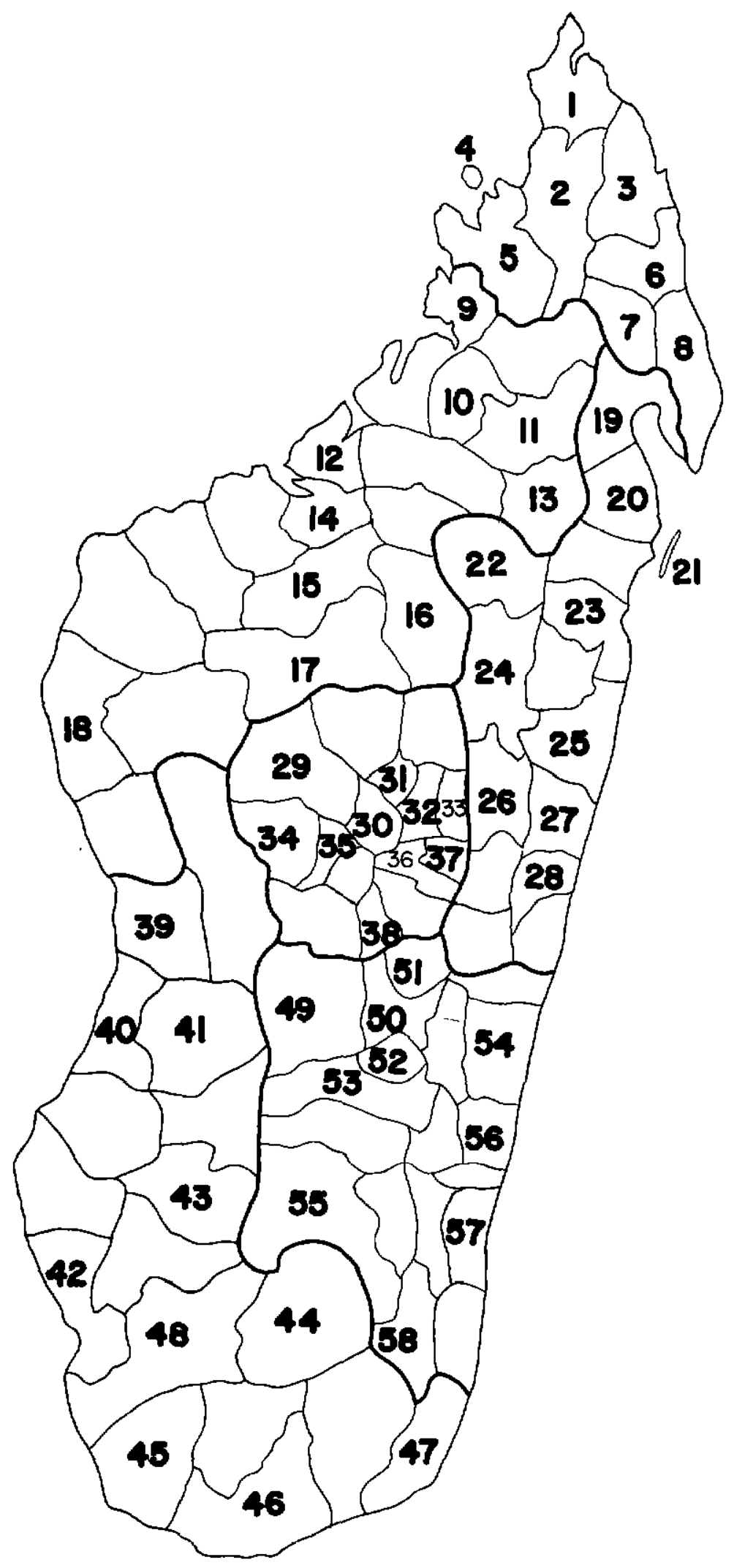


à Madagascar au cours des dix dernières années (incidence générale de la maladie);

- ceux de l'analyse des fiches utilisées au cours de notre enquête (incidence de la maladie par catégorie).

\section{Incidence générale}

\section{de la tuberculose à Madagascar}

Ce relevé concerne les saisies effectuées pour tuberculose à Madagascar au cours des dix dernières années (1958-1968). Nous admettons que la proportion des tuberculeux est celle des saisies pour lésions pulmonaires. La moyenne est calculée sur le nombre d'années pour lesquelles nous avons pu rassembler les statistiques exactes. L'intervalle de confiance est de 1 p. 100 , compte tenu du nombre de relevés étudiés. Eventuellement, nous indiquons aussi la fiabilité de ces résultats compte tenu des sondages de notre enquête. Tous les résultats sont reproduits dans le tableau $n^{0} 1$ (p. 508).

\section{Incidence de la maladie par catégorie de bovins}

Cette incidence ressort directement de l'analyse globale des fiches remplies par les vétérinaires au cours de leurs enquêtes dans les abattoirs : tableau $\mathrm{n}^{\circ} 2$.

\section{TUBERCULINATIONS}

Tous les résultats sont rassemblés dans les tableaux $\mathrm{n}^{\circ} 3$ (zone de Majunga), $\mathrm{n}^{0} 4$ (zone de Morondava), $\mathrm{n}^{\circ} 5$ (zone d'Antsohihy), $\mathrm{n}^{0} 6$ (zone des Hauts-Plateaux) et dans le tableau général $\mathrm{n}^{\circ} 7$ qui rassemble les résultats des quatre zones.

\section{INTERPRETATION ET COMMENT AIRES DE CES RESULTATS}

\section{Tableau no 1}

(incidence générale de la tuberculose par province, au cours des dernières années)

La proportion générale de sujets tuberculeux est donc de 20,83 p. 100 . Bien que ces chiffres ne concernent que le quart environ des animaux abattus chaque année dans l'Ile, ils constituent actuellement les données les plus complètes concernant la maladie. On constate, dans ce tableau, des différences très importantes d'une province à l'autre: elles peuvent concerner des animaux nés dans une autre province, (cas, en particulier, des bovins abattus dans les provinces de Tananarive ou Tamatave), ce tableau ne prétend donc absolument pas être une carte de répartition de la maladie mais un bilan très général de l'enzootie. Une autre variation, qui n'apparaît pas dans ce tableau, concerne les proportions des saisies dans le temps pour une même province : l'analyse de scs causes cst trop complexe pour être tentée dans l'état actuel de nos connaissances. Il est donc difficile d'affirmer que la maladie régresse ou progresse dans certaines zones, compte tenu de l'hétérogénéité des inspections.

TABLEAU $N^{\bullet}$ II

Résultats des inspections d'abattoirs au cours de l'enquête

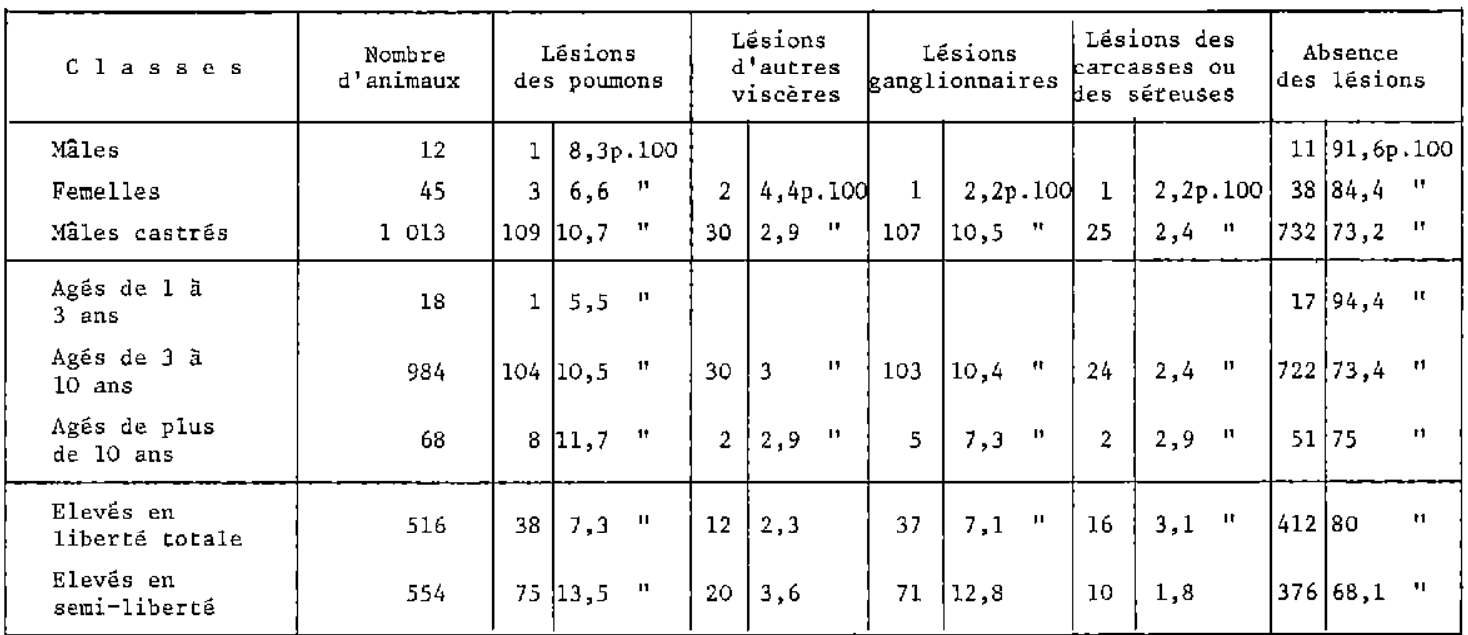




\section{Tableau $n^{\circ} 2$}

Ce tableau concerne les 1.070 carcasses d'animaux inspectées par les vétérinaires au cours de notre enquête : 26 p. 100 de ces animaux présentaient des lésions tuberculeuses.

- Leur fréquence, par catégorie d'animaux est la même que celle démontrée par tubercu- lination, lorsque l'échantillonnage est représentatif.

- Les lésions de la carcasse (ou séreuses pariétales) sont observées dans 2,4 p. 100 des cas. Ce chiffre est à rapprocher de celui constaté par les vétérinaires militaires, de 1957 à $1967: 3,7$ p. 100 de lésions sur les 139.165 carcasses inspectées.

\section{TABLEAU $N^{\circ}$ III}

Proportion de réactions positivea à la tuberculination dans la zone de Majunga (2 241 bovins)

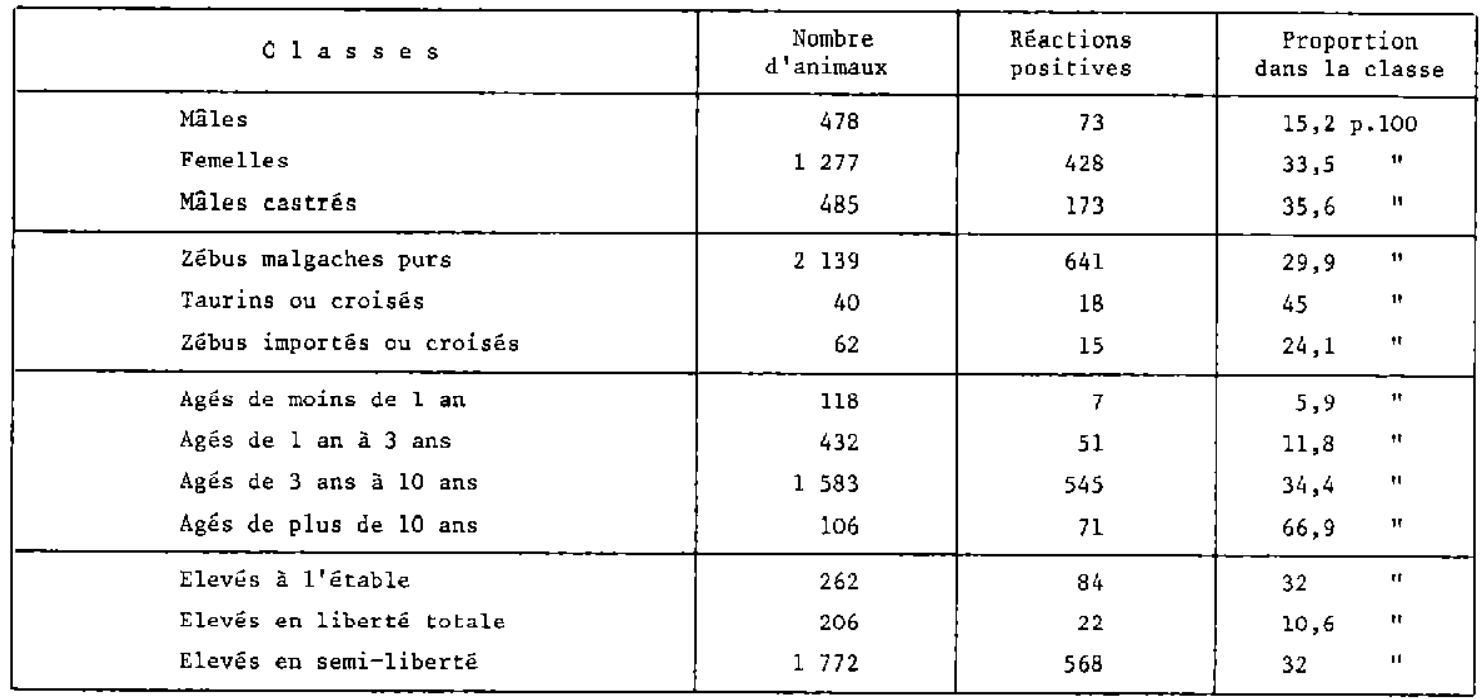

TABLEAU $\mathrm{N}^{*} \mathrm{TV}$

Proportion de reactions positives à la tuberculination dans la zone de Morondava ( 3089 bovins)

\begin{tabular}{|c|c|c|c|}
\hline C I a s s es & $\begin{array}{c}\text { Nombre } \\
\text { d'animaux }\end{array}$ & $\begin{array}{l}\text { Rëactions } \\
\text { positives }\end{array}$ & $\begin{array}{c}\text { Proportion } \\
\text { dans la classe }\end{array}$ \\
\hline Mâles & B14 & 126 & 15,4 p. 100 \\
\hline Femelles & 1576 & 285 & $18 \quad " 1$ \\
\hline Mâles castrẻs & 671 & 167 & 24,8 \\
\hline Zébus malgaches purs & 3073 & 581 & 18,9 \\
\hline Taurins ou croisés & 10 & 1 & 10 \\
\hline Zébus importês ou croisés & 6 & 2 & 33,3 \\
\hline Agés de moins de 1 an & 491 & 40 & 8,1 \\
\hline Agés de 1 an à 3 ans & 657 & 101 & 15,3 \\
\hline Agés de 3 ans à 10 ans & 1138 & 219 & 19,2 \\
\hline Agês de plus de 10 ans & 747 & 212 & 28,3 \\
\hline Elevés à l'étable & 46 & 12 & 26 \\
\hline Elevés en libertê cotale & 1859 & 278 & 14,9 \\
\hline Elevés en semi-libertê & 1156 & 288 & 24,9 \\
\hline
\end{tabular}




\section{TABLEAU $\mathrm{N}^{\circ} \mathrm{V}$}

Proportion de réactions positives à la tuberculination dans la zone d'Antsohihy (1 247 bovins)

\begin{tabular}{|c|c|c|c|}
\hline C 1 a $s$ s e $a$ & $\begin{array}{c}\text { Nombre } \\
\text { d'animaux }\end{array}$ & $\begin{array}{l}\text { Réactions } \\
\text { positives }\end{array}$ & $\begin{array}{l}\text { Proportion } \\
\text { dans la classe }\end{array}$ \\
\hline Mâles & 210 & 15 & $7,1 \mathrm{p}, 100$ \\
\hline Femelles & 643 & 48 & 7,4 \\
\hline Mâles castrés & 387 & 59 & 15,2 \\
\hline Zébus malgaches purs & 1247 & 122 & $9,7 \quad 11$ \\
\hline Taurins ou croisês & - & - & - \\
\hline Zêtuus importés ou croisés & - & - & - \\
\hline Agés de moins de 1 ars & 89 & 4 & 4,4 \\
\hline Agês de 1 an a 3 ans & 279 & 11 & 3,9 \\
\hline Agês de 3 ans à 10 ans & 839 & 103 & 12,3 \\
\hline Agés de plus de 10 ans & 29 & 4 & 13,7 \\
\hline Elevés à l'êtable & - & - & - \\
\hline Elevês en liberté totale & 1239 & 122 & $9,8 \quad "$ \\
\hline Elevës en semi-1ibertë & - & - & - \\
\hline
\end{tabular}

TABLEAU N ${ }^{\circ} \mathrm{VI}$

Proportion de réactions positives à la tuberculination dans la zone des Hauts-Plateaux (2 640 bovins)

\begin{tabular}{|c|c|c|c|}
\hline C I a s g e s & $\begin{array}{c}\text { Nombre } \\
d^{\prime} \text { animaux }\end{array}$ & $\begin{array}{l}\text { Réactions } \\
\text { positives }\end{array}$ & $\begin{array}{l}\text { Proportion } \\
\text { dans la classe }\end{array}$ \\
\hline Mâles & 600 & 79 & $13,1 \mathrm{p}, 100$ \\
\hline Femelles & 632 & 53 & 8,3 \\
\hline Mâles castrés & 1396 & 381 & 27,2 \\
\hline Zébus malgaches purs & 2614 & 509 & $19,4 \quad "$ \\
\hline Taurins ou croisês & 26 & 4 & $15,3 \quad 1 "$ \\
\hline Zébus importês ou croisês & - & - & - \\
\hline Agés de moins de 1 an & 114 & 3 & 2,6 \\
\hline Agês de 1 an à 3 ans & 558 & 40 & 7,1 \\
\hline Agés de 3 ans à 10 ans & 1888 & 457 & 24,2 \\
\hline Agés de plus de 10 ans & 56 & 13 & 23,2 \\
\hline Elevés à l'êtable & 150 & 8 & 5,3 \\
\hline Elevês en libertê totale & 210 & 31 & 14,7 \\
\hline Elevés en semi-1ibertê & 2268 & 474 & 20,8 \\
\hline
\end{tabular}

\section{Tableaux $n^{o s} 3,4,5,6$}

La comparaison inter-zones doit être faite avec prudence compte tenu du choix des troupeaux qui peut n'être pas représentatif de la zone étudiée. D'après nos chiffres, il y aurait alors une différence statistiquement significative entre Majunga (29,8 p. 100 de réagissants) Morondava - Hauts-Plateaux (19,4 p. 100 et $18,5$ p. 100$)$ et Antsohihy (9,7 p. 100).
Il est beaucoup plus intéressant de noter que, à de rares exceptions près, les proportions de tuberculeux par catégorie sont identiques d'une zone à l'autre : géographiquement, la sensibilité à la maladie est invariable.

\section{Tableau $n^{\circ} 7$}

Ce tableau rassemble les résultats des quatre précédents. Nous en avons effectué une analyse 
TABLEAU N"VII

Proportion de réactions positives à la tuberculination pour 1 'ensemble des 4 zoncs (9 217 bovins)

\begin{tabular}{|c|c|c|c|c|}
\hline c 1 a s s e s & $\begin{array}{c}\text { Nombre } \\
d^{\prime} \text { animaux }\end{array}$ & $\begin{array}{l}\text { Réactions } \\
\text { positives }\end{array}$ & \multicolumn{2}{|c|}{$\begin{array}{c}\text { Proportion } \\
\text { daps la classe }\end{array}$} \\
\hline Mâles & 2102 & 293 & \multicolumn{2}{|c|}{13,9 p. 100} \\
\hline Femelles & 4128 & 814 & 19,7 & $"$ \\
\hline Mâles castrés & 2939 & 780 & 26,5 & $"$ \\
\hline Zêbus malgaches purs & 9073 & 1853 & 20,4 & $"$ \\
\hline Tauring ou croisês & 76 & 23 & 30,2 & $"$ \\
\hline Zêbus importés ou croisês & 68 & 17 & 25 & \\
\hline Agés de moing de 1 an & 812 & 54 & 6,6 & $"$ \\
\hline Agés de 1 an à 3 ans & 1926 & 203 & 10,5 & $"$ \\
\hline Agés de 3 ans à 10 ans & 5445 & 1324 & 24,3 & $"$ \\
\hline Agés de plus de 10 ans & 938 & 300 & 31,9 & $"$ \\
\hline Elevês à 1'étable & 459 & 104 & 22,6 & $"$ \\
\hline Elevés en liberté totale & 3514 & 453 & 12,8 & $"$ \\
\hline Elevés en semi-liberté & 5196 & 1330 & 25,5 & $"$ \\
\hline
\end{tabular}

statistique qui a déterminé que toutes les différences dans les proportions de sujets tuberculeux, par catégorie, sont significatives ou hautement significatives, sauf les différences concernant la race et les animaux élevés à l'étable, dont nous ne tiendrons donc pas compte. L'incidence de la maladie varie donc selon:

1. Le sexe: les bœufs sont les plus atteints, puis les vaches et les taureaux.

2. L'âge : l'incidence croît avec l'âge.

3. Le mode d'élevage : le parcage favorise la maladie.

Là encore il faut interpréter prudemment: si, par exemple, les boufs sont les plus atteints, ce n'est pas obligatoirement qu'ils soient plus sensibles mais souvent parce qu'ils sont les seuls animaux du troupeau parqués la nuit (bøufs de trait en particulier). Néanmoins ces observations confirment celles de la plupart des auteurs (5). H. RAKOTO signalait déjà, en 1938 , que 30,8 p. 100 des zébus malgaches parqués réagissaient à la tuberculine contre 12,5 p. 100 des zébus élevés en liberté.

Il faut enfin rappeler qu'il s'agit de résuitats qui sont eux-mêmes la somme des résultats concernant différents troupeaux de différents villages : d'un village à l'autre, il a été donné aux enquêteurs de noter des variations de 3 p. 100 à 30 p. 100 , et de 20 p. 100 à 99 p. 100 d'un troupeau à l'autre.

\section{DISCUSSION}

Nous distinguerons deux parties dans cet important paragraphe :

- l'analyse des causes du développement actuel de l'enzootie;

- l'analyse des solutions possibles pour Ia modifier.

\section{CAUSES DU DEVELOPPEMENT ACTUEL DE L'ENZOOTIE}

Nous n'avons pas l'ambition de les analyser toutes, mais de souligner les causes plus évidentes :

\section{Il existe des causes permanentes de dispersion de l'enzootie}

Ce sont les mouvements de bétail liés aux transactions commerciales. S'il n'existe pas, comme en Afrique, de transhumances régulières, il existe cependant un courant de bétail permanent (souvent à sens unique) dans presque toutes les directions de l'Ile. Ce sont ces mouvements qui ont vaincu inexorablement tous les interdits administratifs contre la maladie de 1905 à 1933.

\section{Il existe des causes permanentes}

de persistance de l'enzootie

dans les troupeaux contaminés

En effet, les éleveurs n'éliminent pas d'eux- 
mêmes leurs animaux tuberculeux et de plus sont contraints très souvent (du fait des vols de boufs ou de la nécessité de protéger les cultures) de laisser en contact animaux malades et animaux sains.

La maladie, étant chronique, effraie moins l'éleveur que les autres maladies infectieuses ou parasilaires. Nous avons interrogé, au cours de cette enquête 115 propriétaires de troupeaux. Tous savaient reconnaître la maladie (toux, anorexie, dyspnée après un effort provoqué intentionnellement). Mais la plupart d'entre eux $(40$ p. 100$)$ renoncent à détecter la maladie à la visite d'achat, se contentant de choisir des sujets jeunes "donc probablement sains ". Et lorsque leurs animaux contractent la maladie, 5 p. 100 seulement disent les destiner à la boucherie, 30 p. 100 les abattre euxmêmes (à l'occasion de fêtes rituelles) et 65 p. 100 les laisser mourir : la majorité des sujets atteints et contagieux est donc conservée dans le troupeau et contamine ses congénères jusqu'à leur mort.

\section{SOLUTIONS POSSIBLES POUR FAIRE REGRESSER L'ENZOOTIE}

Nous ne nous attarderons pas à l'étude d'une régression naturelle possible de la maladie. P. VALETTE (7) pense que cela est possible dans le sud de l'lle, quoiqu'il reste très difficile de comparer des statistiques établies entre 1903 et 1969 , compte tenu de tous les changements administratifs, techniques et commerciaux survenus entre ces deux dates.

Pour l'ensemble du pays, la maladie ne paraît pas régresser. Ce serait à notre connaissance le premier exemple d'un pays où l'enzootie tuberculeuse bovine recule spontanément.

En excluant bien sûr l'expectative totale devant la maladie, il n'y a, selon nous, que deux options possibles actuellement:

- soit transposer progressivement à Madagascar les méthodes de prophylaxie sanitaire qui ont fait leurs preuves en pays d'élevage intensif (Europe, Amérique, Japon, etc.) (3);

- soit adopter une méthode d'éradication particulière pour Madagascar.

Nous envisagerons successivement ces deux options, leurs avantages et leurs inconvénients :

\section{Peut-on transposer à Madagascar} les méthodes d'éradication qui ont réussi en pays d'élevage intensif?

Il s'agit des méthodes types a tuberculination - élimination ". Comme la majorité des vétérinaires qui ont envisagé cette solution, nous pensons que, si cela est possible en milieu d'élevage "interventionnel" (fermes ou ranches privés ou administratifs), cela est totalement exclu actuellement en élevage extensif, d'une part parce que les tuberculinations elles-mêmes sont difficiles, onéreuses et ne peuvent toucher tous les animaux d'une région; d'autre part parce que l'élimination des réagissants se heurterait à plusieurs obstacles :

- psychologiques : voir l'exemple de la prophylaxie de la péripneumonie en Afrique;

— économiques : selon P. VALETTE (7) la maladie fait perdre 760 millions de francs malgaches chaque année. Si cette somme était consacrée à l'indemnisation des propriétaires, elle ne permettrait d'éliminer que 10.000 bovins (soit 4 pour mille des réagissants de toute l'Ile), la valeur des viandes récupérées étant supposée subventionner l'opération (tuberculinations d'élimination et d'achat);

- zootechniques : l'élimination des réagissants déséquilibre le troupeau, qui ne peut être reconstitué que difficilement vu la rareté des sujets indemnes;

- sanitaires: les troupeaux assainis contiendront toujours 3 à 5 p. 100 de sujets anergiques qui les recontamineront aussitôt. Aucune désinfection des pâturages ou abreuvoirs n'est possible.

Ces quatre obstacles ne pourraient être levés qu'au prix de sacrifices disproportionnés par rapport aux pertes actuelles qu'entraîne la maladie ou au danger qu'elle représente pour la santé humaine.

Des variantes "modérées " de la méthode ont été proposées à Madagascar ou dans d'autres pays:

- tuberculiner et isoler les réagissants;

- constituer des régions saines et n'y introduire que des bovins non réagissants;

- éliminer les tuberculeux cliniques, puis les réagissants, en zones limitées puis confluentes. 
Si elles lèvent les critiques sur le plan économique (fractionnement des dépenses), elles ne résolvent pas les autres problèmes, du moins tant que le taux de tuberculose sera aussi élevé à Madagascar.

\section{Peut-on adopter une méthode d'éradication originale propre à Madagascar?}

C'est la solution à laquelle nous pensons. Si l'élevage interventionnel doit continuer pour l'instant à appliquer les méthodes de " tuberculination - élimination ", nous envisagerions plutôt, pour l'élevage extensif uniquement, les procédés suivants :

a) Choix d'une zone expérimentale bien délimitée géographiquement, peu touchée par la maladie et isolée des grands courants commerciaux du bétail;

b) Combinaison, dans cette zone, des procédés de prophylaxie sanitaire et médicale pour abaisser progressivement le taux de bovins tuberculeux.

- Prophylaxie sanitaire : contrôle de l'accès de la zone, celle-ci n'étant autorisée qu'aux animaux de boucherie. Il pourrait être tenté un essai d'élimination des tuberculeux cliniques contagieux, en accord avec les marchands de bestiaux ou les bouchers, qui sont actuellement les seuls à supportcr les pertes financières des saisies.

- Prophylaxie médicale: vaccination annuelle de tous les bovins de la zone choisie, à l'occasion des campagnes de vaccination anticharbonneuse. Un vaccin allergène ne serait pas contre-indiqué, l'épreuve de la tuberculination n'étant plus un critère d'élimination. Si elle le devenait, il suffirait d'attendre un à deux ans que toute allergie vaccinale ait disparu sur l'ensemble des troupeaux.

A Madagascar, les chances de réussite d'une prophylaxie médicale semblent réunies puisque notre enquête démontre que les bovins se contaminent très lentement au cours de leur vie (laissant au vaccin le temps de devancer l'in- fection), que les sujets reproducteurs (vaches, taureaux) sont les moins atteints et que leur mode d'élevage dominant (liberté totale) est défavorable à la maladie.

Seule l'application pratique de la méthode serait délicate et longue, et c'est d'elle que dépendraient en fait les résultats que nous en espérons en théorie : abaissement de l'incidence de la tuberculose bovine à un seuil tel qu'il soit alors possible de faire appel à la seule prophylaxie sanitaire.

\section{CONCLUSION}

Les résultats de cette enquête sur la tuberculose bovine ont actualisé nos connaissances statistiques sur l'étendue de l'enzootie et suggéré les bases scientifiques d'un plan d'éradication. Elle a souligné plusieurs faits qu'il était important de démontrer :

- la difficulté extrême d'une tuberculination intradermique généralisée à l'ensemble des bovins;

- le rôle du mode d'élevage dans l'extension de l'enzootie;

- l'atteinte progressive, mais lente, des sujets au cours de leur croissance;

- l'atteinte relativement réduite des animaux reproducteurs.

Ces faits suggèrent qu'une intervention sanitaire basée uniquement sur la tuberculination puis l'abattage est vouée à l'échec en élevage extensif, mais que sa combinaison avec une méthode de prophylaxie médicale précoce pourrait peut-être soustraire les jeunes à l'infection et abaisser progressivement le taux de tuberculose dans l'ensemble d'un troupeau.

\section{REMERCIEMENTS}

Nous remercions vivement tous les confrères du Service de l'élevage et de l'Office de développement du Moyen-Ouest qui nous ont apporté leur concours dans cette enquête. 
Modèle de fiche d'enquête

\begin{tabular}{|c|c|c|c|c|c|c|c|c|c|c|c|c|c|c|c|c|c|c|c|c|c|}
\hline \multicolumn{3}{|c|}{ Sexe } & \multicolumn{3}{|c|}{ Race } & \multicolumn{4}{|c|}{ Age } & \multicolumn{3}{|c|}{$\begin{array}{c}\text { Mode } \\
\text { d'élevage }\end{array}$} & \multicolumn{2}{|c|}{$\begin{array}{l}\text { Tubercu- } \\
\text { 1ination }\end{array}$} & \multicolumn{2}{|c|}{$\begin{array}{c}\text { Etat } \\
\text { clinique }\end{array}$} & \multicolumn{5}{|c|}{$\begin{array}{l}\text { Lésions } \\
\text { à l'autopsie (1) }\end{array}$} \\
\hline 墨 & 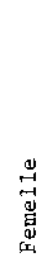 & 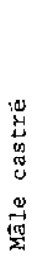 & 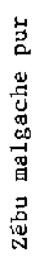 & 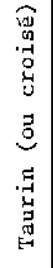 & 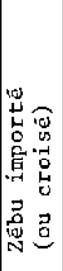 & 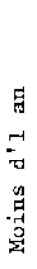 & 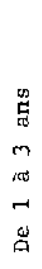 & 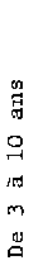 & 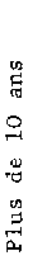 & 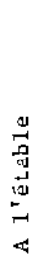 & 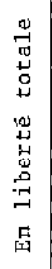 & 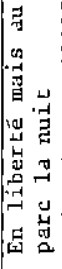 & 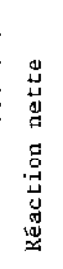 & 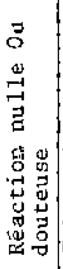 & 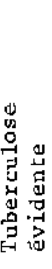 & 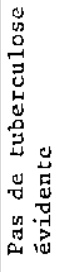 & $\begin{array}{l}5 \\
0 \\
\text { 慁 } \\
\text { 品 }\end{array}$ & 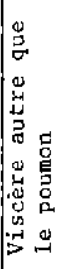 & 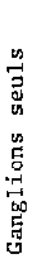 & 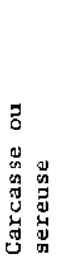 & 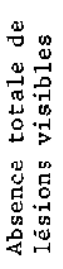 \\
\hline .1 & 2 & 3 & 1 & 2 & 3 & 1 & 2 & 3 & 4 & 1 & 2 & 3 & 1 & 2 & 1 & 2 & 1 & 2 & 3 & 4 & 5 \\
\hline & & & & & & & & & & & & & & & & & & & & & \\
\hline & & & & & & & & & & & & & & & & & & & & & \\
\hline & & & & & & & & & & & & & & & & & & & & & \\
\hline & & & & & & & & & & & & & & & & & & & & & \\
\hline & & & & & & & & & & & & & & & & & & & & & \\
\hline & & & & & & & & & & & & & & & & & & & & & \\
\hline & & & & & & & & & & & & & & & & & & & & & \\
\hline & & & & & & & & & & & & & & & & & & & & & \\
\hline & & & & & & & & & & & & & & & & & & & & & \\
\hline & & & & & & & & & & & & & & & & & & & & & \\
\hline
\end{tabular}

- Nom de L'enquêteur $=$

- Provenance des 10 animaux inspectés (canton) =

- Conment remplir la fiche : I1 y a une rangée pour chacun des 10 bovins : pour chaque animal, mettre une croix dans la case

Toujours grouper les animaux de même provenance sur une même feuille qui lui convient.

(1)S'il y a plusieurs lésions sur le même animal, emplir une seule case : celle de la lésion la plus grave.

\section{SUMMARY}

\section{Bovine tuberculosis in Madagascar}

In 1969-1970, a survey of bovine tuberculosis have been carried out by means of meat inspection findings and tuberculinic tests. The percentage of tuberculosis condemnations in the slaughter-houses of the whole island during last ten years is reported and checked by regional controls: 21 p. 100 of 1.465 .000 cattle inspected had tuberculosis. 9.217 tuberculinic tests have been carried out: their results are given according to race, sex and age of animals. Solutions presently possible to reduce the disease in Madagascar are considered.

\section{RESUMEN}

\section{La tuberculosis bovina en Madagascar}

Se realizó, en Madagascar en 1969-1970, una encuesta sobre la tuberculosis bovina mediante los métodos de inspección de matadero y de tuberculinación intradérmica.

Sondeos regionales permitieron registrar y comprobar la proporción de los descomisos por causa de tuberculosis, efectuados en todos los mataderos de la Isla durante los diez últimos años: Se encontraron tuberculosos 21 p. 100 de los 1.465 .000 bovinos inspeccionados. Se analizaron los resultados de 9.217 tuberculinaciones según raza, sexo, edad y modo de ganaderia de los bovinos comprobados.

Se considera las soluciones actualmente posibles en Madagascar para disminuir la tasa de tuberculosis bovina. 


\section{BIBLIOGRAPHIE}

1. GIDEL (R.), ALBERT (J. R.), RETIF (M.), Enquête sur la tuberculose bovine au moyen de tests tuberculiniques dans diverses régions d'Afrique occidentale (Haute-Volta et Côte d'Ivoire). Résultats et considérations générales, Rev. Elev. Méd. vét. Pays trop., 1969, 22 (3): 337-35.

2. ORUE (J.), CHAMBRON (J.), Rapport sur les tuberculoses animales dans les Etats de l'Afrique noire d'expression françaıse. Leur incidence éventuelle sur la santé humaine, Sixièmes Journées Médicales de Dakar, janvier 1969.

3. RAFYI (A.), La tuberculose animale spécialement étudiée du point de vue des problèmes qui se posent dans les pays en voie de développement, Bull. Off. int. Epiz. 1970, 73 (3-4) : 297-379.

4. RAMON (G.), La lutte préventive contre la tuber- culose. I. Immunité et allergie en matière de tuberculose; II. Cas particulier de la prophylaxie de la tuberculose bovine; vaccination et mesures sanitaires, Bull. Off. int. Epiz. 1951, 35 (3-4): 113-77.

5. Rapports annuels du Service de l'Elevage et du Laboratoire Central de l'Elevage à Madagascar, Tananarive, Archives I.E M.V.T.

6. SCHAFFNER, RAZAFINDRAHABA, La tuberculose à Madagascar. Mise au point de la situation épidémiologique. Points d'impact et perspectives d'avenir, Ann. Univ. Madagascar (Méd.) 1964, 2, V 3: 101-108.

7. VALETTE (P.), La tuberculose animale à Madagascar, Tananarive, Archives du Service de l'Elevage, 1968. 